\title{
Malignant granular cell tumor of the lumbar region - a case report and review of the criteria for diagnosis
}

\author{
Olga Stanowska' ${ }^{1}$ Tadeusz Morysiński², Piotr Rutkowski², Anna Klimczak², \\ Anna Szumera-Ciećkiewicz ${ }^{1,3}$
}

\begin{abstract}
'Department of Pathology and Laboratory Diagnostics, Maria Sklodowska-Curie National Research Institute of Oncology, Warsaw, Poland 2 Department of Soft Tissue/Bone Sarcoma and Melanoma, Maria Sklodowska-Curie National Research Institute of Oncology, Warsaw, Poland ${ }^{3}$ Department of Diagnostic Hematology, Institute of Hematology and Transfusion Medicine, Warsaw, Poland
\end{abstract}

We report an unusual case of a malignant granular cell tumor of the left lumbar region in 63-year old woman - diagnosed, consulted and treated with surgical resection (R1) and radiotherapy, followed up for 2 years with lung metastases after 22 months. Furthermore, we discuss histopathological differential diagnosis and current criteria for malignancy, as well as available options for systemic treatment in view of cytogenetic and molecular genetic characteristics of the tumor.

NOWOTWORY J Oncol 2020; 70, 1:33-36

Key words: granular cell tumor, malignant granular cell tumor, differential diagnosis, immunophenotype

\section{Introduction}

Granular cell tumor (GCT) was first described in 1926 by Abrikossoff as myoblastoma, although it is now believed that tumor cells are of Schwannian origin [1]. Malignant granular cell tumor (MGCT) was first reported in 1945 by Ravich et al. and comprises $0.5-2 \%$ of all GCT cases [2]. The current literature review includes no more than 100 MGCT case descriptions. MGCTs are usually larger and faster-growing than their benign counterparts, the female-to-male ratio is lower and they are more often located in the skin (which can ulcerate) or soft tissue of extremities and trunk rather than head and neck region or gastrointestinal tract. Most importantly, they exhibit metastatic potential. The classification for malignancy is still debatable, and there persists the gray zone, where lesions have the vague potential for local recurrence or distant metastases.

In 1998, Fanburg-Smith et al. proposed subsequent criteria for histopathological MGCT, based on their study of 73 cases: necrosis, at least 3 mitoses per 10 high power fields (HPF, 400 $x$ magnification), pleomorphism, spindling of the tumor cells, increased nuclear to cytoplasmic ratio, vesicular nuclei with prominent nucleoli [3]. According to the suggested criteria, at least 3 out of 6 features are required to confirm the malignancy; 1 or 2 suggest uncertain behavior (atypical GCT); only focal pleomorphism strongly advocates for a benign tumor. Curtis et al. classified MGCT in 3 categories:

1. tumors with both malignant behavior and malignant histology,

2. tumors with atypical histology that are clinically aggressive but not metastatic,

3. tumors with aggressive clinical behavior that are histologically benign [4].

In 2011 Nasser et al. suggested other criteria for malignancy: confirmed metastasis - as being the only accurate - and histological and cytological characteristics (necrosis and/or mitoses present) - only indicative of the malignant potential of the lesion (GCT-UMP) [5].

Due to the rare occurrence of MCGT, regardless of its further biological behavior, the pathologist is obliged to differentiate the lesion from a list of mimickers. For tumors with histologically atypical features, sarcomatoid carcinoma, melanoma, epithelioid malignant peripheral nerve sheath tumor (MPNST), alveolar soft part sarcoma (ASPS), dermatofibrosarcoma (DFSP), angiosarcoma and leiomyosarcoma must be excluded. 
We present a case report of a 63-year old Caucasian female with MGCT (classified according to Fanburg-Smith criteria) in the lumbar region and discuss the classification, differential diagnosis, and treatment.

\section{Case presentation}

A 63-year old female with lumbar pain for 6 months underwent radiographic imaging with magnetic resonance scan revealing soft tissue, hypodense, poorly circumscribed, solid mass of $10 \times 8 \times 8 \mathrm{~cm}$, infiltrating lumbar muscles (Fig. 1 - A.1). The initial diagnosis made by open biopsy sampling outside our center was of Abrikossoff tumor with features suspicious for malignancy and a histopathological consultation was evaluated in the Department of Pathology and Laboratory Diagnostics, Maria Sklodowska-Curie National Research Institute of Oncology in Warsaw.

\section{Pathology findings}

Biopsy showed sheets of spindled, polyhedral and focally pleomorphic cells with abundant, granular, eosinophilic cytoplasm with focal condensations of intracytoplasmic hyaline-like globules and vesicular nuclei with prominent nucleoli. Necrosis was present as well as mitotic activity of 3/10 HPF. Tumor displayed immunopositivity with $\mathrm{S} 100$ (nuclear and cytoplasmic, diffuse and strong), TFE3 (nuclear, strong), SOX10 (nuclear, strong), CD56 (membrane and cytoplasmic, diffuse and strong), CD68KP1 (cytoplasmic, focal, weak), NSE (cytoplasmic, diffuse, weak), Nestin (cytoplasmic and membrane, diffuse, weak) and negative for CKAE1/AE3, SMA, HMB-45, NF, GFAP mono, Inhibin, Calretinin, Desmin, MITF, Melan-A. The pathological findings are presented in figure 1 - A.2.

\section{Treatment and follow-up}

Excision of the tumor was undertaken in the Maria Sklodowska-Curie National Research Institute of Oncology in Warsaw. The excised specimen was non-encapsulated, white-tan, firm, homogenous tumor of $9 \times 8.5 \times 8 \mathrm{~cm}$ with focal necrosis $(5 \%$ of the tumor mass), which was located mostly above and partially under the fascia of lumbar muscle. The margins were involved by the tumor (microscopically $\mathrm{R} 1$ resection). Microscopic examination confirmed the initial diagnosis of malignant granular cell tumor. The patient underwent adjuvant radiotherapy [VHAT with CBCT, 6MV, 30 fractions per 2 Gy, total dose $60 \mathrm{~Gy}$ ] and remains under close observation. After 22 months from the operation the patient developed local recurrence and distant metastases and was referred to regional hospital for chemotherapy (Adriamycin - $15 \mathrm{mg} / \mathrm{m}^{2}$, Dacarbazine - $150 \mathrm{mg} / \mathrm{m}^{2}$, Cyclophosphamide - $100 \mathrm{mg}$ for 5 days every 21 days).

\section{Discussion}

The importance of depicting cases with malignant features lies in the poor prognosis for metastatic disease (60\% survival in 3 years). Due to the low number of cases, guidelines for staging, treatment, and follow-up are still lacking. A wide excisional margin is optimal because of the infiltrative pattern of growth and the tendency to recur. It has been described that MGCTs can result from malignant transformation of benign GCT, so margins preservation is highly recommended also for benign-appearing lesions [6-8].

Macroscopic sampling is one of the key points in diagnostics, especially when the lesion is $4 \mathrm{~cm}$ or larger; following the standard protocol for soft tissue sarcoma processing is advised. Differential diagnosis of the cases with malignant features (necrosis, >2 mitoses/ 10 HPF, high nuclear to cytoplasmic ratio, polymorphism, spindling of the cells, vesicular nuclei with prominent nucleoli) should include melanoma, MPNST, DFSP, spindle cell carcinoma [9]. The broad panel of immunohistochemical stainings is needed. Briefly, melanomas are usually positive for more than one melanocytic markers, i.e. HMB-45, Melan-A, and MITF; MPNST shows weaker and focal expression of $\mathrm{S} 100$ in comparison to GCT/MGCT, DFSP is positive for CD34 and carcinomas more often express cytokeratins. In difficult cases, the panel needs to be extended according to
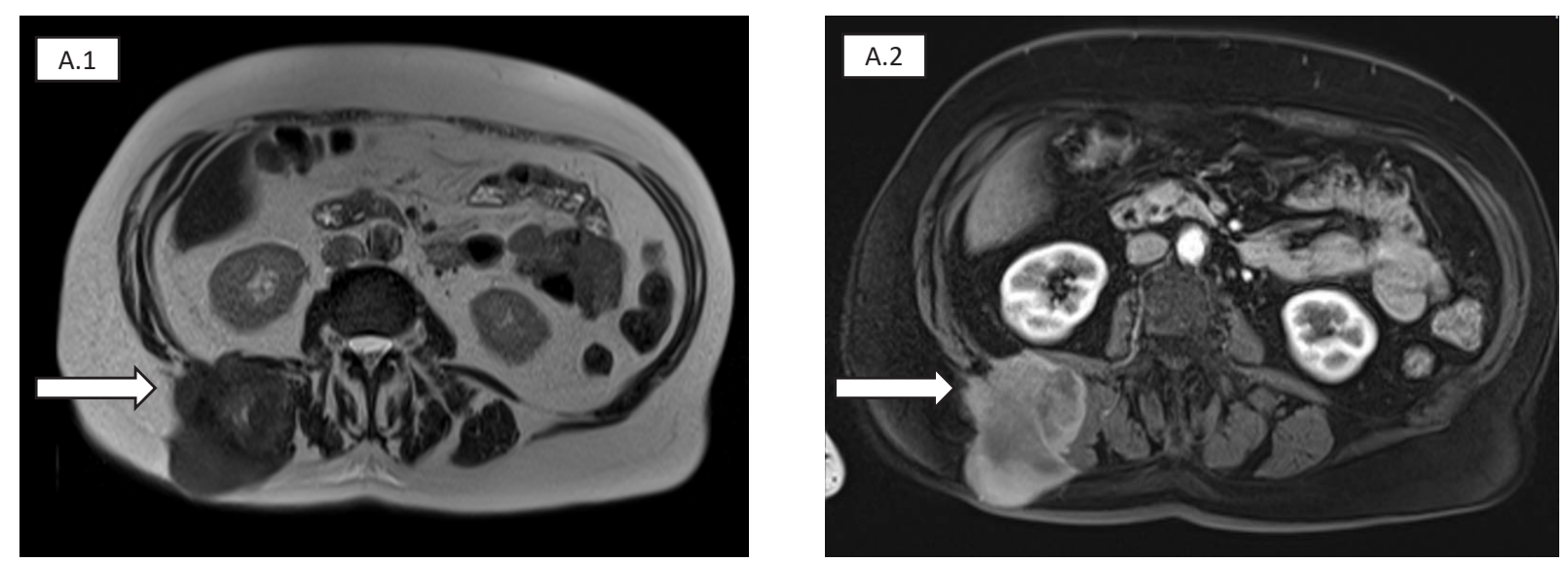

Figure 1. Magnetic resonance scan revealing soft tissue, hypodense, poorly circumscribed, solid mass (arrows) of $10 \times 8 \times 8 \mathrm{~cm}$, infiltrating lumbar muscles (A.1 \& A.2) 
morphological features and results of initial immunophenotypisation. In the presented case, TFE3 was strongly positive, but no PAS/D granules were found, which helped to exclude ASPS. Moreover, the additional "neural" panel of consecutive stains was evaluated including GFAP, NF, NSE, CD56, SOX10, and Nestin; it tends to be positive in MPNST and negative in ASPS [9-11]. The immunohistochemical characteristics with differential diagnosis were depicted in table I.

The pathologist should always highlight the possibility of aggressive behavior, based on recognized histological features (especially necrosis and mitosis) and high Ki-67 ratio (>10\% is a poor prognostic factor). It is debatable if"malignant granular cell tumor" can be a histopathological diagnosis rather than a clinical one (confirmed metastasis) and if a designation of "granular cell tumor with uncertain malignant potential" seems to be more accurate, especially in the setting of rapidly-
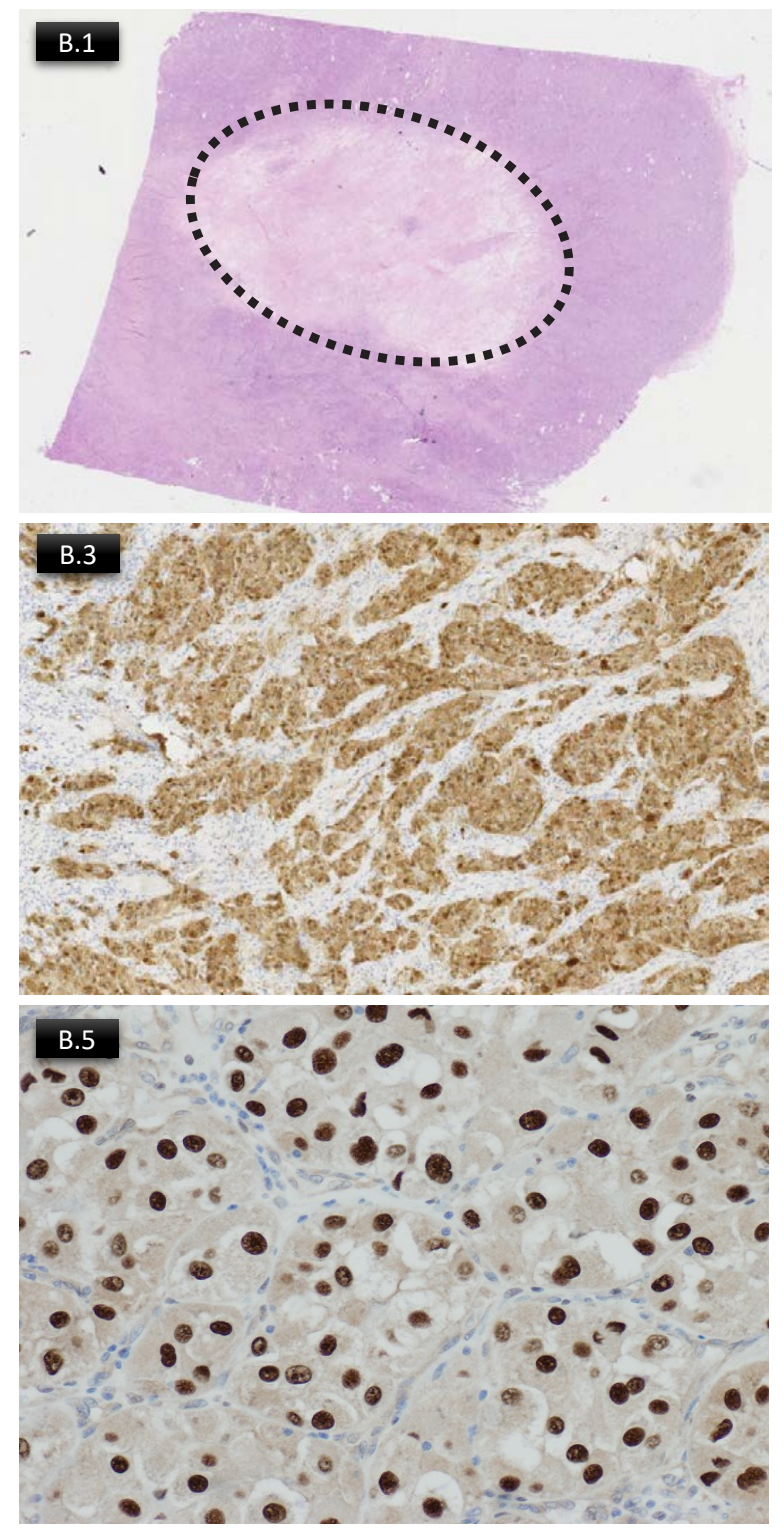

-growing or large tumor (>4 cm). The diagnostic criteria of MGCT according to Fanburg-Smith et al. and Nasser et al. are presented in figure $2[3,5]$.

The diagnosis of GCT-UMP requires continuous observation of the patient. Wide excision margins remain the best possible option, as the role of chemotherapy or radiation therapy remains indefinite. In MGCT, adjuvant radiotherapy on the tumor bed can be delivered with the aim of reducing local recurrence risk [11]. In our case, due to R1 resection of the lesion, the patient underwent postoperative radiotherapy. The two-year follow-up showed aggressive tumor behavior with local recurrence and local metastases.

The results of genetic studies on GCT/MGCT are scarce (based on single cases), but have revealed the heterogeneity of the alterations with no specific karyotype and the absence of most of the alterations described in schwannomas and
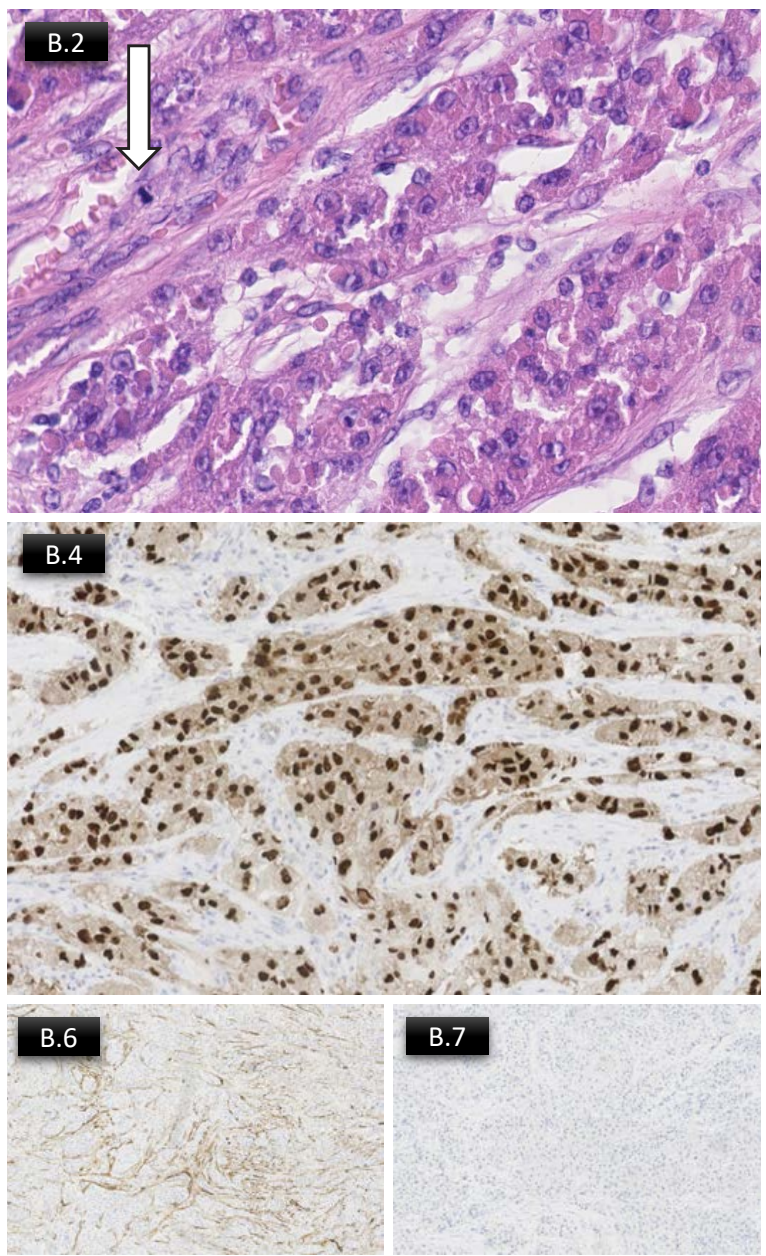

B.7
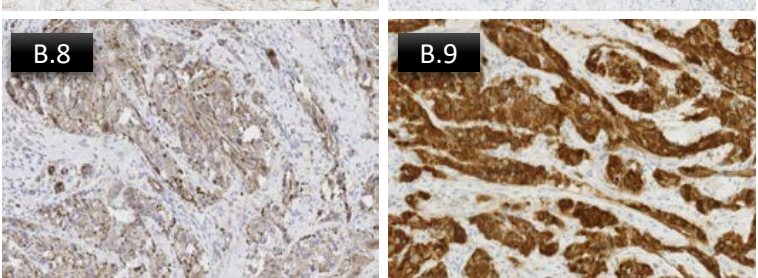

Figure 2. B.1: Hematoxylin and eosin staining ( $\mathrm{HE}, 40 \mathrm{x}$ ) with marked necrosis (circle); B.2: $\mathrm{HE}(200 \mathrm{x})$ with visible mitotic activity (arrow); B.3: $\mathrm{S100}(100 \mathrm{x})$; B.4: SOX10 (100x); B.5: TFE3 (400x); B.6: SMA (100x); B.7: HMB-45 (100x); B.8: Nestin (100x); B.9: CD56 (100x magnification) 
Table I. Immunohistochemical differential diagnosis of granular cell tumor/ / malignant granular cell tumor (GCT/MCGT - granular cell tumor/ malignant granular cell tumor; EMPNST - epithelioid malignant peripheral nerve sheath tumor; ASPS - alveolar soft part tumor; MM - malignant melanoma; CA - cancer); blue - positive, navy - negative, white - positive in limited number of cases

\begin{tabular}{l|l|l|l|l|l|}
\hline \multirow{2}{*}{ Marker } & \multicolumn{5}{c}{ Type of maliganancy } \\
\cline { 2 - 6 } & GCT/MGCT & EMPNST & ASPS & MM & \multicolumn{1}{c|}{ CA } \\
\hline PAS/D & & & & & \\
\hline S100 & & & & & \\
\hline Inhibin & & & & & \\
\hline SOX10 & & & & & \\
\hline Nestin & & & & & \\
\hline Calretinin & & & & & \\
\hline TFE3 & & & & \\
\hline CK & & & & & \\
\hline HMB45 or Melan-A & & & & & \\
\hline CD68 & & & & \\
\hline
\end{tabular}

MPNST. Overall, the sequencing results indicate that the abnormalities of ASXL1-, Notch2-, and PARP4-mediated pathways are possibly involved in the disease initiation and progression of MGCT $[12,13]$. Moreover, single studies showed metabolic response to treatment with pazopanib - a small-molecule inhibitor of vascular endothelial growth factor receptor-1, -2 and -3 , platelet-derived growth factor receptor-a and $-\beta$, and $c-k i t$, which is an approved drug in the treatment of soft tissue sarcomas and there are first reports that this targeted therapy allows for improvement of progression-free survival [13-16].

\section{Conflicts of interests: none declared}

\section{Anna Szumera-Ciećkiewicz}

Maria Sklodowska-Curie National Research Institute of Oncology Department of Pathology and Laboratory Diagnostics ul. Roentgena 5

02-781 Warsaw, Poland

e-mail:szumann@gmail.com

\section{Received: 4 Nov 2019}

Accepted: 11 Dec 2019

This work has been implemented using the Project infrastructure POIG.02.03.00-14-111/13 funded by Operational Programme Innovative Economy 2007-2013, Priority II. R\&D Infrastructure, Measure 2.3. Investments connected with development of IT infrastructure of Science.

\section{References}

1. Abrikossoff A. Über Myome. Virchows Archiv für Pathologische Anatomie und Physiologie und für Klinische Medizin. 1926; 260(1): 215-233, doi: $10.1007 /$ bf02078314.

2. Ravich A, Stout AP, Ravich RA. Malignant Granular Cell Myoblastoma Involving the Urinary Bladder. Ann Surg. 1945; 121(3): 361-372, doi: 10.1097/00000658-194503000-00010, indexed in Pubmed: 17858577.

3. Fanburg-Smith JC, Meis-Kindblom JM, Fante R, et al. Malignant granular cell tumor of soft tissue: diagnostic criteria and clinicopathologic correlation. Am J Surg Pathol. 1998; 22(7): 779-794, doi: 10.1097/00000478199807000-00001, indexed in Pubmed: 9669341.

4. Curtis BV, Calcaterra TC, Coulson WF. Multiple granular cell tumor: a case report and review of the literature. Head Neck. 1997; 19(7): 634-637, doi: 10.1002/(sici)1097-0347(199710)19:7<634::aid-hed12>3.0.co;2-2, indexed in Pubmed: 9323154.

5. Nasser H, Ahmed Y, Szpunar SM, et al. Malignant granular cell tumor: a look into the diagnostic criteria. Pathol Res Pract. 2011; 207(3): 164168, doi: 10.1016/j.prp.2010.12.007, indexed in Pubmed: 21282016.

6. Cui Y, Tong SS, Zhang YH, et al. Granular cell tumor: A report of three cases and review of literature. Cancer Biomark. 2018; 23(2): 173-178, doi: 10.3233/CBM-170556, indexed in Pubmed: 30223384.

7. Jaloux C, Degardin N, Cegarra-Escolano M, et al. A thoracic parasternal granular cell tumor in a child: About one case and review of the literature, discussion about treatment guidelines, is complete resection compulsory? Ann Chir Plast Esthet. 2017; 62(3): 251-254, doi: 10.1016/j. anplas.2016.12.004, indexed in Pubmed: 28104379.

8. Tsukamoto S, Takeda M, Honoki K, et al. Malignant granular cell tumor of the median nerve: a case report with a literature review of 157 cases. Skeletal Radiol. 2019; 48(2): 307-316, doi: 10.1007/s00256-018-3017-2, indexed in Pubmed: 29980826.

9. Fletcher CDM. Organization W.H.WHO Classification of Tumours of Soft Tissue and Bone. IARC Press 2013.

10. Chamberlain BK, McClain CM, Gonzalez RS, et al. Alveolar soft part sarcoma and granular cell tumor: an immunohistochemical comparison study. Hum Pathol. 2014; 45(5): 1039-1044, doi: 10.1016/j. humpath.2013.12.021, indexed in Pubmed: 24746209.

11. Marchand Crety C, Garbar C, Madelis G, et al. Adjuvant radiation therapy for malignant Abrikossoff's tumor: a case report about a femoral triangle localisation. Radiat Oncol. 2018; 13(1): 115, doi: 10.1186/s13014-0181064-4, indexed in Pubmed: 29925410.

12. Xu S, Zhao Q, Wei $S$, et al. Next Generation Sequencing Uncovers Potential Genetic Driver Mutations of Malignant Pulmonary Granular Cell Tumor. J Thorac Oncol. 2015; 10(10): e106-e109, doi: 10.1097/ JTO.0000000000000640, indexed in Pubmed: 26398830.

13. Wei L, Liu S, Conroy J, et al. Whole-genome sequencing of a malignant granular cell tumor with metabolic response to pazopanib. Cold Spring Harb Mol Case Stud. 2015; 1(1): a000380, doi: 10.1101/mcs.a000380, indexed in Pubmed: 27148567.

14. Conley A, Koplin S, Caracciollo J, et al. Dramatic Response to Pazopanib in a Patient With Metastatic Malignant Granular Cell Tumor. J Clin Oncol. 2014; 32(32): e107-e110, doi: 10.1200/jco.2012.47.1078.

15. Morita S, Hiramatsu M, Sugishita M, et al. Pazopanib monotherapy in a patient with a malignant granular cell tumor originating from the right orbit: A case report. Oncol Lett. 2015; 10(2): 972-974, doi: 10.3892/ ol.2015.3263, indexed in Pubmed: 26622607.

16. Graaf Wv, Blay JY, Chawla S, et al. Pazopanib for metastatic soft-tissue sarcoma (PALETTE): a randomised, double-blind, placebo-controlled phase 3 trial. Lancet. 2012; 379(9829): 1879-1886, doi: 10.1016/s01406736(12)60651-5. 